\title{
The Effect Of Culture System On Embryonic Development and Aneuploidy Rate For Icsi Cases \\ Yasmine Sayed Azouz ${ }^{1}$,Mohamed Abbas Eid ${ }^{2}$, Mohamed Refaat Shehata ${ }^{3}$,Heba Ali Abd EL-Rahman ${ }^{4}$ \\ 1.Ganin Fertility Center, Cairo, Egypt ,2.Andrology and Sexology - Faculty of Medicine - Cairo University,3. Inorganic Chemistry , 4. Zoology Department ,Faculty of Science - Cairo University \\ Corresponding author:Yasmine Sayed Azouz,email:Yasminsayed32@gmail.com,mobile:00201154682010
}

\begin{abstract}
Background: Patients undergoing intracytoplasmic sperm injection (ICSI) or in vitro fertilization (IVF)cycles often suffer from recurrent implantation failure (RIF). To ensure live birth and embryonic viability, culture systems should be optimized in all ICSI and IVF cycles. The success of IVF can be affected by the culture systems employed for embryonic development. It has been argued that covering micro drops with oil in culture systems can prevent microbial infection, ensure suitable osmotic and $\mathrm{pH}$ conditions, and prevent the evaporation of the medium.
\end{abstract}

Objectives: Comparing the embryonic development, euploidy rate , and clinical outcome of using GPS Dishes covered with paraffine oil and SPL Dishes with mineral oil for patients with previous RIF.

Patients and methods: the study was a prospective randomized controlled trial and the sample size consisted of 174 patients undergoing ICSI cycles at a private fertility center during the period between April 2017 and March 2020. Patients' embryos were randomly assigned into one of the two of the aforementioned groups.

Results: The two groups weren't statistically significantly different in male age, female age, collected oocyte number, number of mature oocytes, and number of blastocysts undergoing preimplantation genetic diagnosis (PGD-A). For the preimplantation parameters of the embryos, fertilization, cleavage, and high-quality blastocyst rates were statistically significantly higher in the GPS Dishes with paraffin oil group than in the SPL Dishes covered with mineral oil group (77.5, 80.1 , and $79.45 \%)(p=0.012$, and 0.001$)$.

Conclusion: we conclude that embryonic development can be enhanced by using GPS Dishes with paraffin oil overlying.

Keywords: ICSI; RIF; Culture systems; IVF media; Embryo development.

\section{INTRODUCTION}

Couples suffering from infertility problems caused by a severe male factor or those who underwent a previous in vitro fertilization (IVF) without successful fertilization are recommended to seek intracytoplasmic sperm injection (ICSI) treatments. ICSI can be helpful to patients who have low chances of conceiving as it bypasses various fertilization barriers ${ }^{(1)}$.

To ensure the delivery of healthy babies and the viability of embryos, the culture systems in IVF laboratories should be ideal. The success of IVF can be affected by the culture systems employed for embryonic development ${ }^{(2)}$. Typically, media used in IVF are covered with paraffin or mineral oils to ensure the stability of the media during embryonic development. Oils can help to slow down the processes of variations in $\mathrm{pH}$ and temperature as well as evaporation. Embryonic development can be adversely affected by increased media osmolality which can result from the absence of an oil overlay ${ }^{(3)}$.

For optimum ART and embryo development outcomes, IVF laboratories should concentrate on reducing stressors with detrimental effects on cells in IVF laboratories. This is of crucial importance due to the sensitivity of the oocytes, spermatozoa, and embryos ${ }^{(4)}$. Several elements within IVF laboratories can act as negative stressors on cells including different chemical and mechanical factors (FIGURE 1). 


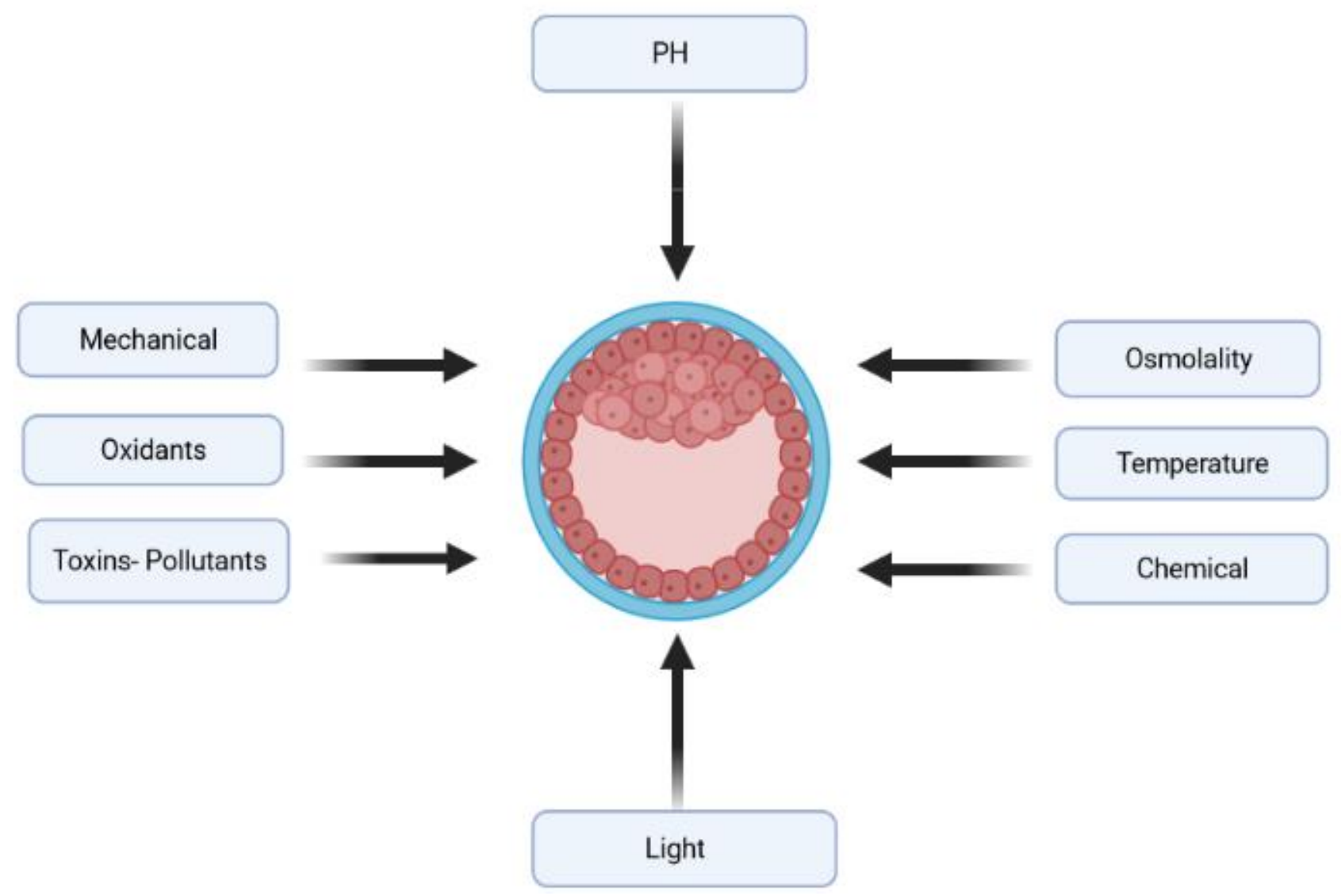

Figure 1:stressors typically present in culture media that can adversely affect the process controlling for proper chromosomal separation/segregation of chromosomes and mitotic cell division as well as in vitro embryonic development.

The cellular machinery in embryos can be directly affected by factors present in the culture media such as antioxidants and metabolic substrates. In addition, embryonic cells can be adversely affected by negative stressors created by the degradation of compounds in culture media. Therefore, it is recommended to assess the effect of culture media components on embryo quality ${ }^{(5)}$.

To prevent adverse effects of increased osmolality and evaporation over time, several factors should be taken into consideration such as the quantity and type of the oil cover, medium volume, and type of dish used (6). Furthermore, the $\mathrm{pH}$ of the media can be raised due to extra stressors caused by evaporation ${ }^{(7)}$.

Aneuploidy or the abnormal number of chromosomes in embryos may be affected by various factors such as $\mathrm{O}_{2}$ concentration, osmolality, temperature, $\mathrm{pH}$, and culture media. Aneuploidy is the chief culprit of birth defects, recurrent pregnancy loss, failure of implantation, and embryo arrest ${ }^{(8)}$.

Selecting and transferring viable and healthy embryos can affect the effectiveness of in vitro fertilization (IVF). Morphology of the embryos was assessed under the microscope for selection purposes (morphology assessment) ${ }^{(9)}$. Nevertheless, not all embryos showing normal morphology can lead to the delivery of a healthy baby.

This study aims to compare the pre-implantation embryonic parameters as well as the clinical outcomes between using paraffin oil with GPS dishes or mineral oil with SPL dishes culture system methods for patients experiencing ICSI treatment.

\section{MATERIALS AND METHODS Study design}

The sample size consisted of 174 couples undergoing ICSI treatments in Ganin Fertility Center. For eligibility criteria, all females over 37 and less than 18 years old were excluded, all females had to have at least 5 metaphase II oocytes to be used for injection. Based on the semen parameters references by WHO 2010, only couples whose male partners had normal seminal fluid analysis parameters were included.

\section{Controlled ovarian stimulation and oocyte pick up}

All the female subjects received a long ovulation stimulation protocol combined with oral contraceptive pills Gynera ${ }^{\circledR}$ (Bayer, Leverkusen, Germany) from the third day of the menstrual cycle till 12-21days with the 
last five days overlapped with $20 \mathrm{IU}$ of Lucrin ${ }^{\circledR}$ (Abbvie, Madrid, Spain). Transvaginal ultrasound and an endocrine assessment from the third day of the following cycle were performed to ensure the downregulation. In case of confirmation, a 150 IU Fostimon ${ }^{\circledR}$ (IBSA, Collina d'Oro, Switzerland) or Merck Serono, Rome, Italy) along with 75 IU Menopur ${ }^{\circledR}$ (Ferring, Kiel, Germany) was used to induce ovarian stimulation. Throughout the next days, such dosages were adjusted to suit the response of each patient. A 10,000 IU of hCG (Pregnyl®; Organon, Oss, The Netherlands) was instantly injected when a minimum of two follicles was observed to have $\geq 19 \mathrm{~mm}$ diameter.

Thirtysix hours post the injection of hCG, female subjects were anesthetized generally, and with the aid of ultrasound-guided transvaginal, follicles were aspirated, and oocytes were collected. A multipurpose handling medium MHM (Irvine Scientific, CA, USA) supplemented with $10 \%$ serum substitute supplement (SSS; Irvine Scientific) was used for the retrieval of Cumulus-oocyte complexes (COCs). 50 $\mu \mathrm{l}$ drops of continuous single culture (CSC; Irvine Scientific) medium supplemented with $10 \%$ SSS were used for washing and culturing of the COCs till the time of denudation and injection.

\section{Sperm preparation}

Participants were instructed to abstain from sexual activities for 1 to 2 days. Semen samples were attained by ejaculation and were left to liquefy for 30 minutes. This was followed by microscopic assessment of semen parameters: sperm morphology, motility, and count, based on the WHO (2010) references for normal sperm parameters.

Implemented techniques of sperm preparation and selection were previously tested to ascertain that the study outcomes were not adversely affected. Density gradient centrifugation was used for sperm preparation by adding $1 \mathrm{ml}$ of liquefied semen to a $15-\mathrm{ml}$ conical tube containing a $50 \%$ and $90 \%$ discontinuous double-layer Isolate $\AA$ (Irvine Scientific).

Centrifugation of samples was performed for 8 minutes at $250 \times \mathrm{g}$ and at room temperature. The supernatant was discarded and a Sperm wash medium (Irvine Scientific) was added to the pellet and mixed and then the mixture was centrifuged $250 \times \mathrm{g}$ for $8 \mathrm{~min}$ at room temperature. The supernatant was discarded and the remaining pellet was used in later sperm selection techniques.

\section{ICSI, embryo culture}

2 to 4 hours after oocyte retrieval, an embryologist injected the mature oocytes which were then cultured into either of the two culture systems of the study's groups.
For the first group, GPS dishes were used by the addition of 25- $\mu$ l drops of NX media supplemented with $10 \%$ SSS (Serum Substitute Supplement) overlayed with paraffin oil which was loaded before media loading, and the cases were incubated at $37{ }^{\circ} \mathrm{C}, 5 \% \mathrm{O}_{2}$, and $6 \% \mathrm{CO}_{2}$. In the second group, SPL dishes were used with a mineral oil overlay which was added after the loading of media. And also the cases were incubated at $37{ }^{\circ} \mathrm{C}, 5 \% \mathrm{O}_{2}$, and $6 \%$ $\mathrm{CO}_{2}$.

Gardner's criteria 1990 were used to assess the rates of fertilization, blastocyst formation, and high-quality blastocysts. BB, BA, AB, AA embryos were considered high-quality embryos irrelevant of their degree of expansion. The percentage of total day 5 or 6 blastocysts by the number of the total cleaved embryos was calculated as the blastocyst formation rate.

PGT-A testing was performed by obtaining a trophectoderm biopsy of day 5 or 6 blastocysts and running it on the Next Generation Sequencing technique (MiSeq machine), sequenced byVeriSeq kit, and amplified by SurePlex amp kit. PGT-A by Cooper surgical, San Diego, CA 92122, United States, was used to validate the results.

\section{Statistical Analysis}

For the main finding, a difference in blastocyst formation rate between the two groups was used to determine the calculation of sample size.to maintain the ability to exclude the null hypothesis, 140 patients had to be recruited. The version 3.0.11 of the PS Power and Sample Size Calculations software, version for Microsoft Windows was used for the calculation. The 22 version of the IBM SPSS (Statistical Package for the Social Sciences; IBM Corp, Armonk, NY, USA) for MS Windows was used for statistical calculations. Findings were reported as either percentages, cases number/frequencies, or as mean \pm standard deviation (mean $\pm \mathrm{SD}$ ).

The chi-square $\left(\chi^{2}\right)$ test was used to compare the two groups' categorical variables whereas the numerical data were compared using the Student t-test for independent variables. Statistical significance was considered if the $p$ value was 0.05 or less.

\section{Ethical Approval}

The Center Directorate of Research \& Health Development located in Cairo, Egypt has approved to conduct the current study, research ethical committee in accordance with the ethical standards of the institutional and/or national research committee and with the 1964 Helsinki Declaration and its later amendments or comparable ethical standards. For recruitment, participants had to give written consent. 


\section{RESULTS}

Table 1 Patient characteristics

\begin{tabular}{|l|c|c|c|}
\hline & $\begin{array}{c}\text { SPL dishes, } \\
\text { Mineral oil } \\
\mathbf{n = 8 0}\end{array}$ & $\begin{array}{c}\text { GPS dishes, } \\
\text { Paraffin oil } \\
\mathbf{n = 9 4}\end{array}$ & $\begin{array}{c}\boldsymbol{P} \text { - } \\
\text { value }\end{array}$ \\
\hline Female age (years) & $29.8 \pm 4.29$ & $30.4 \pm 4.46$ & 0.310 \\
\hline Retrieved oocytes & $18.6 \pm 9.51$ & $19.3 \pm 11.4$ & 0.978 \\
\hline MII oocytes & $15.2 \pm 8.32$ & $14.9 \pm 9.84$ & 0.567 \\
\hline Trial outcomes & $77.8 \pm 17.1$ & $83.3 \pm 17.2$ & 0.012 \\
\hline $\begin{array}{l}\text { Fertilization } \\
\text { rate(\% } \pm \text { SD) }\end{array}$ & $75.1 \pm 17.5$ & $80.1 \pm 18.2$ & 0.025 \\
\hline $\begin{array}{l}\text { Cleavage rate } \\
(\% \pm S D)\end{array}$ & $67.9 \pm 19.1$ & $61.8 \pm 22.2$ & 0.053 \\
\hline $\begin{array}{l}\text { Blastocyst } \\
\text { rate(\% } \pm \text { SD) }\end{array}$ & $69.1 \pm 20.1$ & $79.4 \pm 20.3$ & 0.001 \\
\hline $\begin{array}{l}\text { High quality } \\
\text { Blastocyst rate } \\
(\% \pm S D)\end{array}$ & $53.0 \pm 33.9$ & $63.5 \pm 31.3$ & 0.046 \\
\hline $\begin{array}{l}\text { Euploidy rate } \\
(\% \pm S D)\end{array}$ & & & \\
\hline
\end{tabular}

Data presented as mean $\pm \mathrm{SD}$. $\mathrm{n}$ is the number of patients, MII is the metaphase II oocytes, p-value represents the comparison between the two groups. $\mathrm{p} \leq 0.05$ is considered significant.

The two groups were not statistically significantly different in female age, collected oocyte number, number of mature oocytes, and number of blastocysts undergoing PGD-A. For the pre-implantation parameters of the embryos, fertilization, cleavage, and high-quality blastocyst rates, all were statistically significantly higher in the GPS dishes with paraffin oil group than in the SPL dishes covered with the mineral oil group $(77.5,80.1$, and $79.45 \%)(p=0.012$, and 0.001$)$.

The format (mean \pm standard deviation) was used for data presentation, in addition to the p-value which represents the statistical difference between the two groups. If the p-value was 0.05 or less, it was considered statistically significant. Unless mentioned, data were reported in percentages(\%) and numbers.

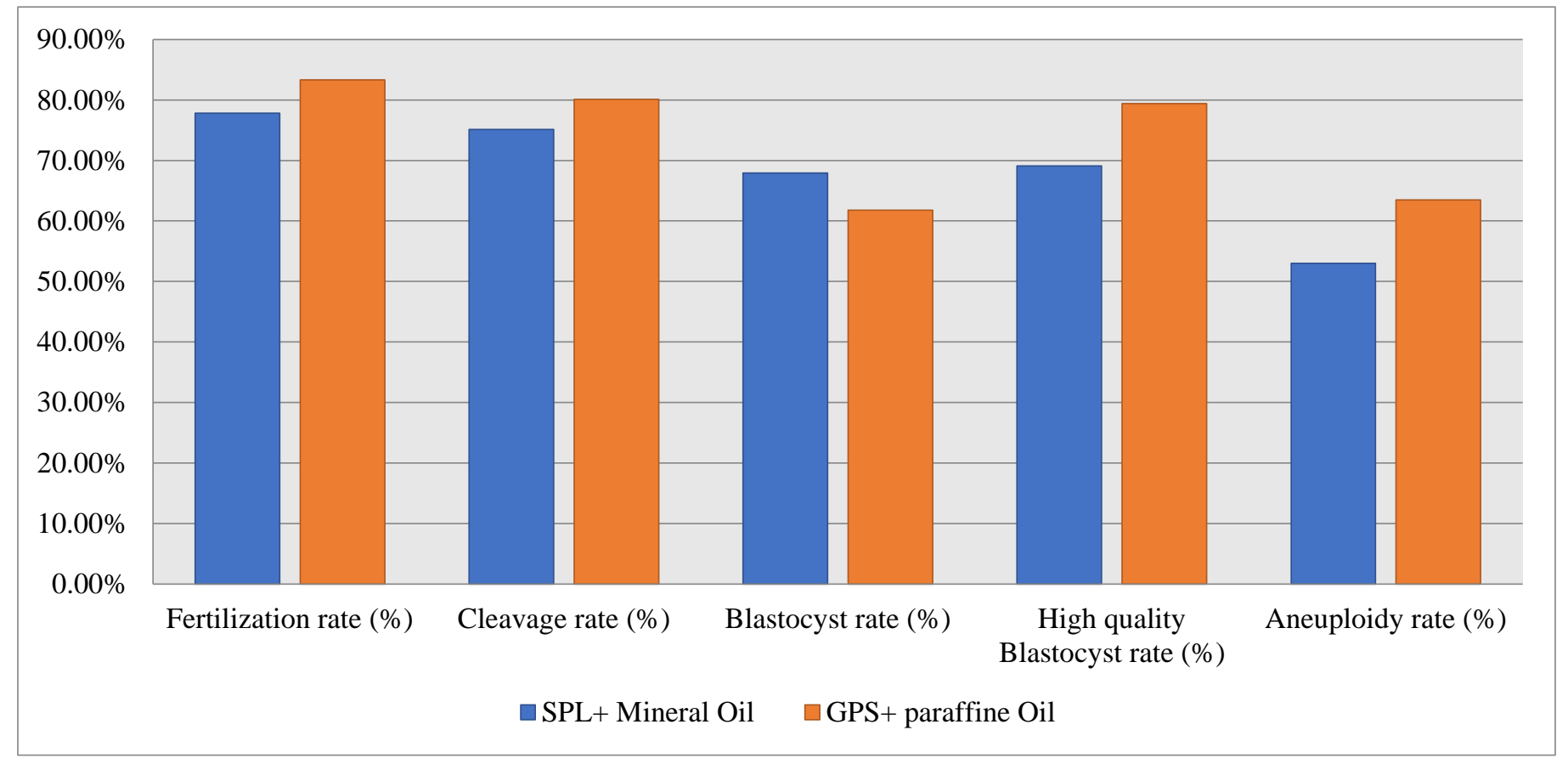

Figure 2: Embryological results of the two groups in the rates of fertilization, cleavage, blastocyst, high-quality blastocyst, and euploidy. High-quality blastocyst rates were statistically significantly higher in the GPS dishes with paraffin oil group than in the SPL dishes covered with mineral oil group 

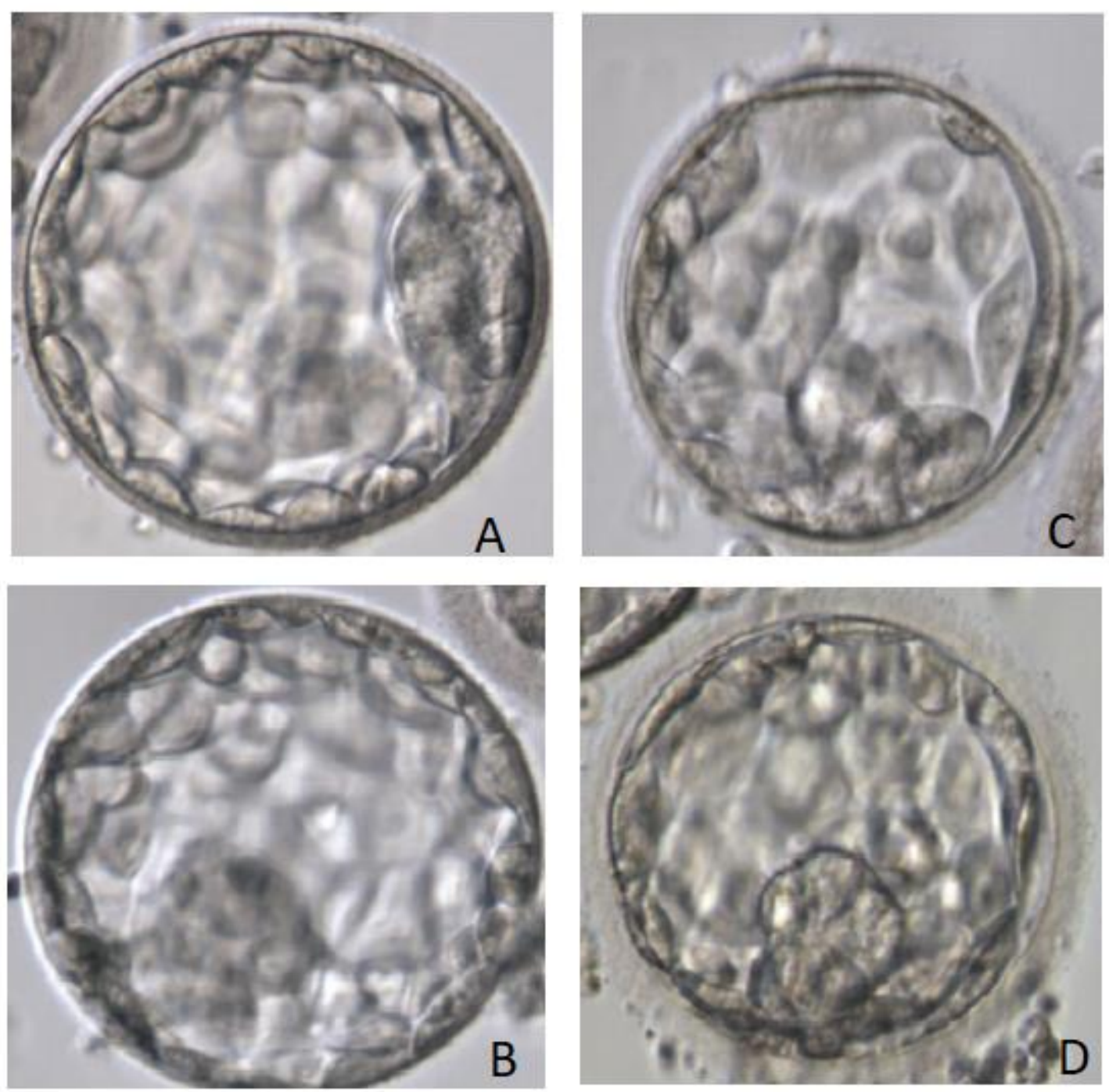

Figure 3: A and B represent a 4AA blastocyst developed from (GPS + paraffin oil) culture system. C and D represent a 4BB and $4 \mathrm{AB}$ blastocyst developed from (SPL + mineral oil) culture system.

\section{DISCUSSION}

Female age, male age, retrieved oocyte number, number of mature oocytes, and number of blastocysts that underwent PGT-A were not significantly different between the two study's groups ${ }^{(10)}$. However, the GPS dishes with paraffin oil overlay group showed significantly higher rates of fertilization, cleavage, and high-quality blastocysts and lower rates of aneuploidy embryos than the SPL dishes with mineral oil overlay group. The blastocyst formation rate was not significantly different between the two groups ${ }^{(11)}$.

Tae et. al (2006) compare the difference between using sterile-filtered light paraffin oil or washed mineral oil on in vitro embryonic development and follicular oocytes in bovine. It was reported that the type of oil used continuously affected in vitro embryo development, this effect, however, was not visible in fertilization or cleavage stages but it started from the morula stage in favor of the group of sterile-filtered light paraffin oil overlying showing qualitative and quantitative improvements ${ }^{(12)}$. Their results were in line with our observations.

Findings showed that the mineral oil group had significantly lower rates $(p<0.05)$ of embryonic development from day 6 (blastocyst and morula) compared to the paraffin oil group (21.7\% vs. $32.8 \%)$ and ( $30.6 \%$ vs. $44.8 \%)$, respectively.

On the other hand, one study with a prospective design ( $n=1237$ sibling oocytes) examined the effect of $100 \%$ mineral and paraffin oils on the embryonic development and rates of day 3 embryos utilization rate (13). The authors reported that live birth rates and embryonic development were not statistically significantly different between the two groups. However, our results showed a statistically significant difference between the two groups with respect to the embryonic development. 
https://ejhm.journals.ekb.eg/

There is limited data on the in vitro physical requirements of embryos during development such as culture dishes which are not typically designed for in vitro embryonic development.

One modification of the conventional Petri dish enables the close gathering of cultured embryos by including small round bottom wells. Such a fashioned dish can prevent micro drops problems including aggregation or shifting of drops, also it facilitates cell recovery and identification. the Embryo Corral dish is a type of GPS dish in which vertical shafts divide every well into four partitions, this enables the sharing of a common media reservoir, and at the same time facilitates embryo observation by allowing the culture of a single embryo in each partition ${ }^{(14)}$.

Gravity may take a while to act on centrally settling the embryos at the bottom of the concave microwell. Oil can either be underlying or overlying the $\mu$ Drop GPS $\AA$ dish. To facilitate the identification of cultured oocytes or embryos, each quadrant in the $\mu$ Drop GPS ${ }^{\circledR}$ dish is marked with a series of numbers and letters. A number from 1 to 12 is molded in every micro-well and a letter from $A$ to $D$ is molded in every quadrant. These markers enable easy tracking and identification of embryonic development.

Couples who can transmit structural abnormalities of the chromosomes or single-gene disorders to the offspring can decrease these odds by using pre-implantation genetic diagnosis (PGD) to select and transfer healthy embryos (15).

The embryos undergoing a biopsy overall $71.8 \%$ were designated to have a broad diagnosis-affected or unaffected embryo consistent with the genetic disorder studied, $70.8 \%$ in the ICSI groups. In addition, it was reported that the percentage of embryos showing positive diagnosis for a certain genetic disorder in the ICSI groups were $36.2 \%$ and $37.3 \%$ in general ${ }^{(15)}$. Hence, the authors recommended the use of ICSI for insemination in PGD cycles.

Patients undergoing ICSI or IVF cycles often suffer from Recurrent implantation failure (RIF). The current study has a prospective design and aims to help people with advanced age and RIF by determining the effectiveness of next-generation sequencing (NGS) based PGT-Aof blastocysts' trophectoderm biopsy on their clinical outcomes. The study recruited 265 couples who suffered from RIF in previous cycles and underwent 250cycles of PGT-A and 346 cycles of follicle aspiration and they were divided into two groups, those in which the female partner is 38 years old or older, and those in which the females are less than 38 years old ${ }^{(16)}$. Findings showed a significantly lower aneuploidy rate in the embryos of the younger group compared to the older group (39.9 vs. 68.9 $\%, P<0.001)$. However, rates of miscarriage ( 7.8 vs. 4.3 $\%$ ), implantation (51.0 vs. $39.1 \%$ ), clinical pregnancy
(48.0 vs. $39.1 \%$ ), and pregnancy (64.7 vs. $43.5 \%)$, per embryo transfer (ET) were not significantly different between the two groups.

Findings showed that RIF can be affected by factors related to the embryo. Patients with advanced age and RIF can benefit from trophectoderm genetic testing using NGS-based PGT-A as it may have positive effects on their clinical outcomes. The current RIF management can be improved by incorporating NGS-based PGT-A. However, additional randomized controlled trials are needed to support this conclusion.

\section{CONCLUSION}

To ensure live birth and embryonic viability, culture systems should be optimized in all ICSI and IVF cycles. Hence, we conclude that embryonic development can be enhanced by using GPS dishes with paraffin oil overlying.

\section{ACKNOWLEDGMENT}

I am greatly grateful to acknowledge Prof. Dr. Mohamed Abbas Eid, Professor of Andrology and Sexology - Faculty of Medicine - Cairo University, for his valuable supervision and continuous encouragement. Many thanks for his kind patience, his valuable scientific advice, and for critically reviewing the manuscript. I am very lucky to have such a great opportunity to be one of his many students.

I can't be thankful enough for Prof. Dr. Mohamed Refaat Shehata, Inorganic Chemistry - DepartmentFaculty of Science - Cairo University for his scientific guidance, his great support, and his very constructive critique. I am very grateful and I consider myself lucky to have him as a supervisor.

My sincere gratitude and deep appreciation for Dr. Heba Ali, Lecturer of Comparative Anatomy and Embryology, Faculty of Science, Cairo University, for supporting extremely good research facilities, constructive supervision, and for her kind comments on the draft manuscript.

My deepest gratitude to all members of the GANIN IVF center for their help.

I owe a special debt with gratitude to my family for their constant support and encouragement. Without you all, I would have never made it.

\section{REFERENCES}

1- Stephens M, Arnett M, Meacham B (2013): The use of in vitro fertilization in the management of male infertility: What the urologist needs to know. Reviews in urology, 15(4):154.

2- Simopoulou M, Sfakianoudis K, Rapani A et al. (2018): Considerations regarding embryo culture conditions: from media to epigenetics. In vivo, 32(3):451-460.

3- Di Lemma L, Davies R, Ford K et al. (2019): Responding to Adverse Childhood Experiences: An evidence review of interventions to prevent and address adversity across the 
life cours. Public Health Wales; Public Health Collaborating Unit Bangor Institute for Health and Medical Research, School of Health Sciences, Bangor University, 1-45.

https://research.bangor.ac.uk/portal/files/23440237/Respo ndingToACEs_PHW2019 english.pdf.

4- Feuer S, Rinaudo P (2012): Preimplantation stress and development. Birth Defects Research Part C: Embryo Today: Reviews, 96(4): 299-314.

5- Wale L, Gardner K (2016): The effects of chemical and physical factors on mammalian embryo culture and their importance for the practice of assisted human reproduction. Human reproduction update, 22: 2-22.

6- Swain E (2019): Controversies in ART: can the IVF laboratory influence preimplantation embryo aneuploidy?. Reproductive biomedicine online, 39(4): 599-607.

7- Swain E (2015): Optimal human embryo culture. In Seminars in reproductive medicine,Thieme Medical Publishers, 33(02): 103-117.

8- Olds S, Stemm K, Wachter K et al. (2015): Analysis of embryo culture media $\mathrm{pH}$ changes during incubator use and media evaporation under oil using a continuous $\mathrm{pH}$ monitoring system. Fertility and sterility, 104(3): e318e319.

9- Revelli A, Canosa S, Carosso A, Filippini C et al .(201 9). Impact of the addition of Early Embryo Viability Assessment to morphological evaluation on the accuracy of embryo selection on day 3 or day 5: a retrospective analysis. Journal of ovarian research, 12(1): 1-7.

10- Esteves C, Carvalho F, Bento F, Santos J (2019): A novel predictive model to estimate the number of mature oocytes required for obtaining at least one euploid blastocyst for transfer in couples undergoing in vitro fertilization/intracytoplasmic sperm injection: the ART calculator. Frontiers in endocrinology, 10: 99.

11- Suzumori N, Sugiura-Ogasawara M (2010): Genetic factors as a cause of miscarriage. Current medicinal chemistry, 17(29): 3431-3437.

12- La Marca A, Capuzzo M, Sacchi S et al (2020): Comparison of euploidy rates of blastocysts in women treated with progestins or GnRH antagonist to prevent the luteinizing hormone surge during ovarian stimulation. Human Reproduction, 35(6): 1325-1331.

13- Tae C, Kim Y, Lee D, Park P, Lim H (2006): Sterile filtered paraffin oil supports in vitro developmental competence in bovine embryos comparable to co-culture. Journal of assisted reproduction and genetics, 23(3): 121127.

14- Rojas A (2021): Socioecological Predictors of Microbiome Variation in Wild Populations of African Mammals (Doctoral dissertation, Michigan State University).https://www.proquest.com/openview/d9bd2d7 3e01931fc2fb227eebea31a8e/1?pqorigsite $=$ gscholar $\& \mathrm{cbl}=18750 \&$ diss $=y$

15- Stern J (2014): Preimplantation genetic diagnosis: prenatal testing for embryos finally achieving its potential. Journal of clinical medicine, 3(1): 280-309.

16- Tong J, Niu Y, Wan A, Zhang T (2021). Next-Generation Sequencing (NGS)-Based Preimplantation Genetic Testing for Aneuploidy (PGT-A) of Trophectoderm Biopsy for Recurrent Implantation Failure (RIF) Patients: a Retrospective Study. Reproductive Sciences, 28(7): 19231929.

17- Feldman B, Aizer A, Brengauz M, Dotan Ket al. (2017): Pre-implantation genetic diagnosis - should we use ICSI for all?. Journal of assisted reproduction and genetics, 34(9): 1179-1183. 